\title{
Respuesta hidrológica de una cuenca de meso escala frente a futuros escenarios de expansión forestal ${ }^{1}$
}

\author{
Mauricio Aguayo ${ }^{2}$, Alejandra Stehr ${ }^{3}$ y Oscar Link ${ }^{4}$
}

\begin{abstract}
RESUMEN
A nivel global más de la mitad del agua generada a través del proceso de escorrentía es usada por el hombre. En el centro-sur de Chile las cuencas hidrográficas han sido sometidas a intensas transformaciones territoriales, incluyendo la forestación masiva con especies exóticas. Este estudio evalúa el efecto hidrológico de futuros escenarios de expansión forestal en una cuenca de meso escala $\left(4.340 \mathrm{~km}^{2}\right)$ utilizando el modelo SWAT. Los escenarios fueron construidos a través del modelo de regresión logística proyectando el desarrollo forestal hacia los años 2018, 2028 y 2038. Los resultados muestran una tendencia a la disminución de los caudales, especialmente en la época de estiaje. Para algunos escenarios la disminución de los caudales de verano supera el $50 \%$ respecto al periodo base. Esta tendencia decreciente de los caudales, producto del aumento de la cobertura forestal, es consistente con la reportada por otros autores en cuencas de pequeña y meso-escala.
\end{abstract}

Palabras clave: cambio de uso del suelo, plantaciones forestales exóticas, producción hídrica, centro-sur de Chile, modelo hidrológico SWAT.

\begin{abstract}
A global level more than half of the water generated through the process of runoff is used by man. In south-central Chile the watersheds have been subjected to intensive processes of land use changes, including expansion of forest plantations with exotic species. This study evaluates the hydrological effects of future scenarios for forest expansion in a basin of mesoscale $(4.340 \mathrm{~km} 2)$ using the SWAT model. The scenarios were constructed through logistic regression model forest development planning to the years 2018, 2028 and 2038. The results of the validation process show that the model has good capacity to represent the hydrology of the watershed under analysis. Simulations indicate a decreasing trend in flows especially in the dry season. For some scenarios, decreasing summer flows exceed $50 \%$ respect to the base period. This decreasing trend is consistent with those reported by other authors in basins of small and meso-scale.
\end{abstract}

Key words: Land use change, forest exotic plantations, water yield, south-central Chile, SWAT hydrological model.

Los autores agradecen a la Comisión Nacional de Investigación Científica y Tecnológica (CONICYT) por el financiamiento recibido a través del proyecto FONDECYT 1120897. Artículo recibido el 25 de mayo de 2015, aceptado el 30 de julio de 2015 y corregido el 24 de septiembre de 2015. Departamento de Planificación Territorial / Facultad de Ciencias Ambientales/ Centro EULA-Chile, Universidad de Concepción (Chile). E-mail: maaguayo@udec.cl

3 Departamento de Ingeniería Ambiental / Facultad de Ciencias Ambientales/ Centro EULA-Chile, Universidad de Concepción (Chile). E-mail: astehr@udec.cl

4. Departamento de Ingeniería Civil / Facultad de Ingeniería, Universidad de Concepción (Chile). E-mail: olink@udec.cl 
Para todo aquel involucrado en hidrología y, en general, en el manejo de recursos hídricos, el cambio de uso del suelo es un problema que no podrá evitar (Sahin \& Hall, 1996). A nivel global, más de la mitad del agua generada a través del proceso de escorrentía es usada por el hombre (Postel et al., 1996). Las propiedades biogeofísicas del territorio tales como cobertura de suelo, rugosidad, relieve y tipos de suelo son determinantes en la generación de la escorrentía. Así por ejemplo, un cambio en la cobertura vegetal, generado por diversas prácticas de uso del suelo, puede alterar significativamente el balance de agua superficial y la partición de las precipitaciones dentro de los procesos de evaporación, escorrentía, y flujo de agua subterránea (Sahin \& Hall, 1996, Foley et al., 2005). Los efectos hidrológicos generados por cambios en el uso del suelo están fuertemente relacionados con la cuantía, distribución y posibilidades de aprovechamiento de los recursos hídricos (Echeverría et al., 2008, Mark \& Dickinson, 2008).

La respuesta hidrológica de cuencas de pequeña escala $\left(<10 \mathrm{~km}^{2}\right)$ ante cambios en la cobertura del suelo se encuentra ampliamente estudiada y sus, impactos, bien documentados (Bosch \& Hewlett, 1982; Sahin \& Hall, 1996; Brown et al., 2005; Huber et al., 2008; Lara et al., 2009). Sin embargo, la literatura sugiere que en cuencas de meso y gran escala $\left(>10 \mathrm{~km}^{2}\right)$ los efectos no son tan obvios (Wilk et al., 2001). Las magnitudes de los cambios de cobertura del suelo requeridos para observar variaciones en los procesos hidrológicos no han sido bien establecidas y depende tanto de las características biogeofísicas de la cuenca como de las condiciones de borde previas al evento de lluvia (Bronstert et al., 2002). Según Klöcking y Haberlandt (2002), la respuesta hidrológica de grandes cuencas ante cambios en el uso del suelo están determinadas por la reacción individual de sus subcuencas. Mecanismos compensatorios tales como desincronización de los flujos "peak" y capacidad de almacenaje del paisaje se convierten en factores importantes en cuencas de gran escala. De esta manera, los efectos del uso del suelo sobre el proceso de escorrentía y generación de caudal, fuertemente determinados por la magnitud y localización de los cambios, parecen ser más relevantes a nivel de subcuencas; por lo tanto, la evaluación de los impactos deben ser realizados, necesariamente, a través de un análisis espacialmente explícitos y temporalmente distribuido (Klöcking \& Haberlandt, 2002).

Los modelos hidrológicos se han transformado en una herramienta útil para analizar las consecuencias del cambio de uso del suelo sobre los componentes que determinan el régimen hidrológico (Fohrer et al., 2001). Estos son capaces de simular la dinámica del agua en los diferentes compartimentos del balance hídrico en función, por ejemplo, de diferentes tipos de cobertura vegetal y características del suelo incluyendo una descripción de los principales flujos verticales y laterales que se suceden en una cuenca hidrográfica (Klöcking et al., 2003). La evaluación de los impactos generados por el cambio de uso del suelo, a través del uso de modelos hidrológicos, se centra en determinar cómo estos cambios alteran el régimen de caudal (Fohrer et al., 2001; Bronstert et al., 2002, Eckhardt et al., 2003). En esta dirección, el uso eficiente de los recursos hídricos se encuentra fuertemente vinculado a un adecuado entendimiento de los procesos de precipitación/escorrentía y sus efectos de mediano y largo plazo en el balance hídrico (Fohrer et al., 2001). La modelación de los impactos hidrológicos generados por distintos escenarios de uso del suelo permite brindar información confiable y pertinente al proceso de toma de decisiones relacionado con la gestión sustentable del agua a escala de cuenca hidrográfica (Klöcking et al., 2003).

La mayoría de las investigaciones realizadas en Chile hacen referencia a los efectos hidrológicos del reemplazo de praderas y bosque nativo por plantaciones forestales a escala de microcuencas (por ejemplo, Huber et al., 2008, Lara et al., 2009). Los escasos estudio realizados en 
cuencas de meso o gran escala ponen en evidencia el potencial impacto que tiene la expansión forestal sobre la escorrentía superficial (Little et al., 2009; Iroumé \& Palacios, 2013). En este sentido, es importante considerar que la principal fuente de abastecimiento hídrico del centro-sur de Chile corresponde a cursos de agua superficial cuya recarga depende del régimen de precipitaciones. Muchas de las cuencas que dan origen a estos cuerpos de agua han sido sometidas a intensos cambios en el uso del suelo incluyendo la tala de bosque nativo, el desarrollo de actividades agropecuarias y, en las últimas décadas, la forestación masiva con especies exóticas de rápido crecimiento, fuertemente subsidiada por el estado a partir de la entrada en vigencia del Decreto Ley No 701 en 1974 (Aguayo et al., 2009; Lara et al., 2012; Altamirano et al., 2013). Este acelerado proceso de forestación ha hecho surgir con fuerza la percepción de que las plantaciones forestales disminuyen notablemente la producción hídrica de cuencas con régimen pluvial. La reducción de la disponibilidad de agua asociada con la forestación a gran escala ha estado generando una creciente preocupación en la población (Iroumé \& Palacios 2013). Los problemas de abastecimiento de agua son cada vez más frecuentes en cuencas que proveen de este recurso a comunidades rurales, sobre todo en los meses de verano (Iroumé et al., 2005, Iroumé \& Palacios, 2013).

En este contexto, las preguntas de investigación que se abordan en este trabajo se centran en responder cómo y en qué medida el incremento de las plantaciones forestales afectará al régimen de caudal en una cuenca de meso escala localizada en el centro-sur de Chile. Se hipotetiza que el aumento masivo de plantaciones forestales provoca una reducción significativa de la escorrentía superficial y, en consecuencia, del caudal. El objetivo de este estudio es evaluar la respuesta hidrológica de futuros escenarios de expansión forestal en la cuenca del río Vergara, subcuenca del río Biobío, usando técnicas de modelación hidrológica espacialmente distribuida. A partir de los resultados, se pretende proporcionar antecedentes objetivos que orienten la planificación y toma de decisiones hacia una gestión integrada y sustentable de cuencas hidrográficas en Chile.

\section{Metodología}

La cuenca del río Vergara se encuentra ubicada entre $37^{\circ} 29^{\prime}-38^{\circ} 14^{\prime} \mathrm{S}$ y $71^{\circ} 36^{\prime}-73^{\circ} 20^{\prime} \mathrm{O}$ y forma parte del sistema hídrico de la cuenca del río Biobío (Figura № 1). Posee una superficie de $4.340 \mathrm{~km}^{2}$ y presentan un clima templado cálido con temperaturas que varían entre los $18^{\circ} \mathrm{C}$ en enero y los $8^{\circ} \mathrm{C}$ en junio. La precipitación promedio anual es de $1.650 \mathrm{~mm}$. Los caudales medios máximos y mínimos ocurren durante los meses de julio-agosto (estación húmeda) y febrero-marzo (estación seca), respectivamente. Las elevaciones fluctúan entre los 200 y $1.900 \mathrm{~m}$. Los suelos poseen texturas franco-limosas en su parte alta y franco-arcillosas en la parte media/baja de la cuenca. Los usos de suelo más importantes corresponden a terrenos agropecuarios, plantaciones forestales y vegetación nativa (Aguayo et al., 2008; Stehr et al., 2010).

\section{Modelo hidrológico}

El modelo hidrológico SWAT (Soil and Water Assesment Tool, versión SWAT2012) y su interfaz gráfica ArcSWAT fue usado para evaluar los potenciales efectos de la expansión forestal en la cuenca. SWAT es un modelo semi-distribuido que fue desarrollado para predecir el impacto de distintas prácticas de uso de suelo sobre el balance hídrico y la exportación de sedimentos y 
agroquímicos en cuencas complejas de meso y macro escala (Arnold et al., 1998, Fohrer et al., 2001, 2005, Arnold \& Fohrer, 2005). La necesidad de información espacial para SWAT incluye un modelo digital de elevación y mapas de tipos y usos de suelo. En este estudio se utilizó el modelo digital de elevación SRTM (Shuttle Radar Topography Mission), las series de suelo presentes en la cuenca y descritas en los estudios agrológicos de las regiones del Biobío y Araucanía (CIREN 1999a y 1999b), y los usos del suelo generados a partir de imágenes Landsat ETM+ y Spot 5 para los años 2000 y 2008, respectivamente. Toda la información cartográfica fue procesada en formato raster con una resolución de 30 metros. Para la discretización espacial de la cuenca fue necesario delimitar subcuencas, las cuales a su vez fueron subdividas en unidades de respuesta hidrológica (URHs). Las URHs son unidades dentro de las cuales existen condiciones relativamente homogéneas de uso y tipo de suelo, por lo que se espera que estas unidades presenten un comportamiento hidrológico igualmente homogéneo. Los procesos hidrológicos simulados incluyen escorrentía superficial, infiltración, flujo lateral subsuperficial, flujo de agua subterránea y evaporación potencial (Arnold et al., 1998, Arnold \& Fohrer, 2005). Debido a la falta de valores locales para los parámetros que describen las características hidrológicas de los diferentes usos del suelo presentes en el área de estudio, estos fueron asociados a la base de datos disponible en el modelo SWAT (Stehr et al., 2010). En el caso particular de las plantaciones forestales, estas fueron consideradas como plantaciones adultas de Pinus radiata y Eucaliptus spp. plenamente establecidas.

Para los propósitos de calibración del modelo se utilizó series de tiempo de precipitaciones, temperaturas y caudales comprendidas entre el año 2000 y 2002. La cobertura de uso del suelo usada en la modelación de caudales corresponde a la del año 2000. El método de Curva Número (CN) fue utilizado para el cálculo de la escorrentía debido a la disponibilidad de datos de precipitaciones y caudales diarios. El rastreo de la escorrentía fue modelada utilizando el método de onda cinética y la ecuación de Manning para determinar las velocidades de escurrimiento. Para la recarga de acuíferos subterráneos SWAT utiliza la función de decaimiento exponencial propuesta por Venetis (1969) modificada por Sangrey et al. (1984). Los cálculos de evapotranspiración fueron realizados usando el método de Hargreaves, el cual requirió de una serie diaria de temperaturas máximas y mínimas. El conjunto de datos meteorológicos fue obtenido desde 16 estaciones de precipitación y tres estaciones de temperatura localizadas alrededor de la cuenca del río Vergara. El set de datos de caudales fue obtenido desde tres estaciones fluviométricas correspondientes a las subcuencas Renaico (678 km2), Mininco (427 km2) y Malleco (401 km2) (Figura No 1). Los resultados del análisis de sensibilidad y proceso de calibración del modelo para la cuenca del río Vergara se encuentra detallados en Stehr et al. (2010). En general, el modelo reproduce satisfactoriamente el orden de magnitud de los caudales observados y su tendencia en el tiempo; sin embargo, el modelo subestima los caudales máximos (Stehr et al., 2010).

El modelo fue validado comparando los caudales medios mensuales simulados versus los caudales medios medidos en las estaciones fluviométrica asociadas a las subcuencas de Renaico, Mininco y Malleco durante los años 2002-2008. El nivel de ajuste entre los caudales simulados y observados fue evaluado a través de los índices: 1) eficiencia de Nash-Sutcliffe (EF), 2) valor absoluto del porcentaje de desviación (PBIAS), y 3) coeficiente de determinación $\left(\mathrm{R}^{2}\right)$ según los rangos de calificación definidos por Van Liew et al. (2005) (Cuadro No 1). 
Figura No 1

Localización geográfica de la cuenca del río Vergara y estaciones fluviométricas de Renaico, Mininco y Malleco
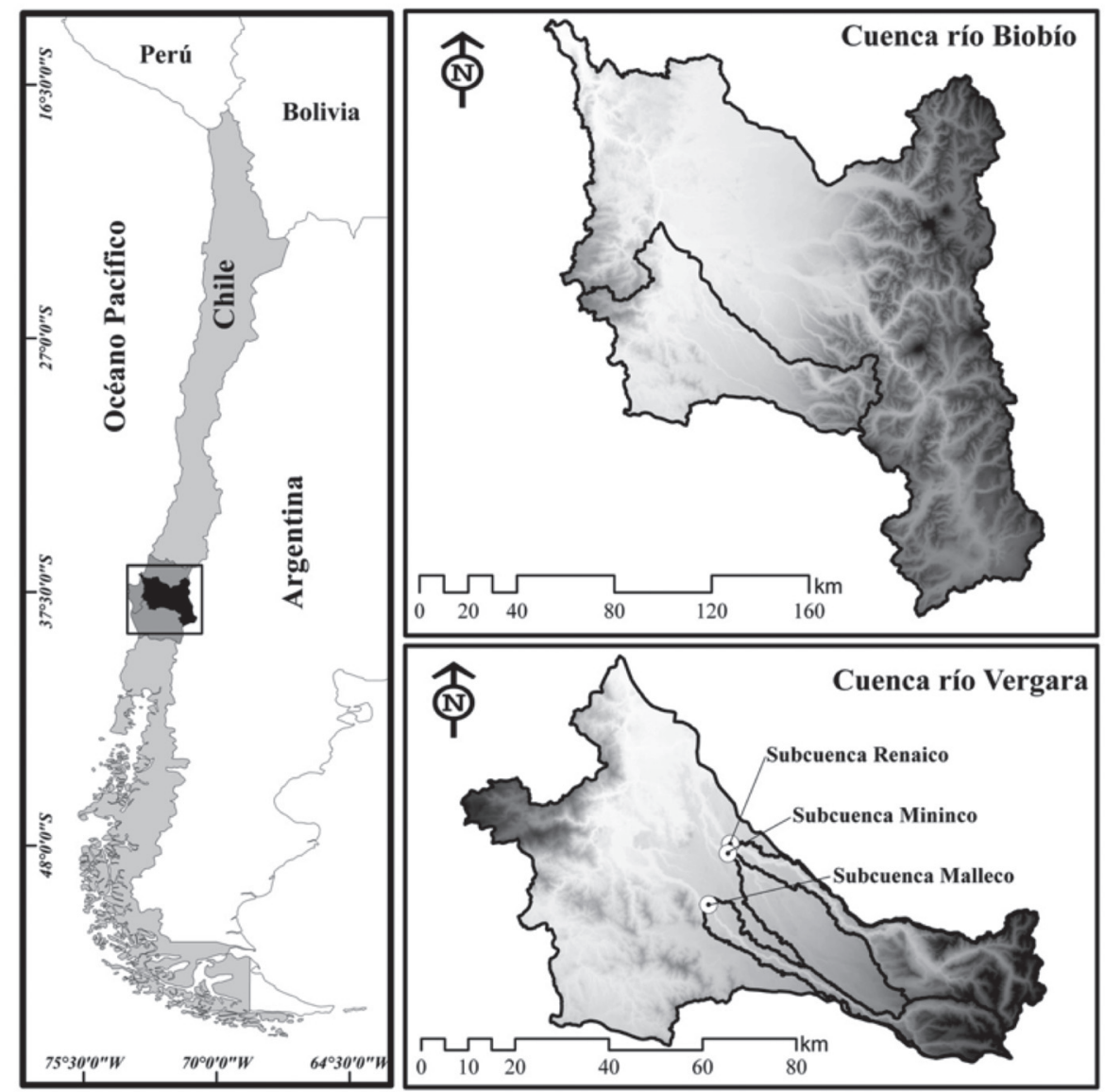

Fuente: Elaboración propia.

Cuadro No 1

Calificación del proceso de calibración y validación

\begin{tabular}{|l|l|l|}
\hline \multicolumn{1}{|c|}{ Índices } & \multicolumn{1}{|c|}{ Rangos } & \multicolumn{1}{c|}{ Calificación } \\
\hline EF & $>0,75$ & Bueno \\
& $0,75-0,36$ & Satisfactorio \\
& $<0,36$ & No Satisfactorios \\
PBIAS & $<20 \%$ & Bueno \\
& $20 \%-40 \%$ & Satisfactorio \\
& $>40 \%$ & No Satisfactorios \\
\hline
\end{tabular}

Fuente: modificado de Van Liew et al. (2005). 


\section{Simulación de escenarios de expansión forestal}

La construcción de escenarios futuros de expansión forestal se basó en la dinámica de los cambios en el uso de suelo ocurridos en la última década. Las coberturas de uso del suelo fueron derivadas a partir de la clasificación de dos imágenes satelitales cuyas escenas corresponden a los años 2000 (Landsat ETM+) y 2008 (Spot 5). El método de clasificación supervisada fue empleado para el procesamiento de las imágenes satelitales. El criterio estadístico de máxima verosimilitud fue elegido para construir la firma espectral que permitió clasificar las imágenes según las categorías de coberturas del suelo previamente definidas (Chuvieco, 1996). A partir del análisis de la dinámica del uso de suelo durante periodo 2000-2008 se identificaron las variables que, a priori, fueron consideradas como factores que determinan el establecimiento de las plantaciones forestales en la cuenca; entre ellas: elevación, pendiente, suelo, uso del suelo, caminos, cuerpos de agua, centros poblados, tamaño de propiedad. La relación entre el patrón espacial de la expansión forestal (variable dependiente) y los "factores forzantes" (variables independientes) fue establecida y cuantificada a través del ajuste del modelo de regresión logística. Usando el método stepwise fueron seleccionadas las variables que aportan, significativamente, a la descripción del patrón espacial del avance de las plantaciones forestales en la cuenca (Aguayo et al., 2007). Los parámetros del modelo de regresión logística fueron usados para simular escenarios de expansión forestal con intervalos de 10 años; esto es, 2018, 2028 y 2038. La simulaciones suponen que la tasa de expansión forestal y las variables que determinan su localización geográfica no varían en el tiempo. El análisis y modelación de los escenarios fueron realizados a través de los software ArcGIS 9.1 e IDRISI Kilimanjaro utilizando datos en formato raster con tamaños de celda de 30 metros.

\section{Simulación de respuesta hidrológica}

El modelo SWAT calibrado y validado fue usado para simular los caudales medios mensuales durante los periodos 2015-2020, 2025-2030, y 2035-2040, consideran los escenarios de expansión forestal 2018, 2028 y 2038, respectivamente. La simulación de los caudales medios supone que el régimen de precipitaciones y temperaturas registradas durante el periodo de calibración y validación se mantienen invariante en el tiempo. Esto implica que la disminución de precipitaciones y el aumento de temperaturas proyectados para la región, según distintos escenarios de cambio climático, no fueron considerados (CONAMA-DGF 2006). En este sentido el único factor que generaría variaciones en la hidrología de la cuenca serían los escenarios de expansión forestal simulados. De esta manera, para evaluar el efecto del aumento de la cobertura de plantaciones forestales sobre el régimen de caudal, se compararon los datos simulados con los datos medidos en las estaciones fluviométricas de Renaico, Mininco y Malleco durante los años 2005-2010 (período base). La comparación se realizó cuantificando las variaciones porcentuales de los caudales medios mensuales de las épocas de verano (enero-marzo) e invierno (julio-septiembre).

\section{Cambios de uso de suelo y futuros escenarios de expansión forestal}

Los principales cambios en el uso del suelo ocurridos en la cuenca durante el periodo 2000 2008 se deben, principalmente, al acelerado avance de las plantaciones forestales (Figura $N^{\circ} 2$ ). En el transcurso de diez años la cobertura de plantaciones forestales aumentó 42,7 mil hectáreas 
llegando a ocupar cerca del $50 \%$ de la superficie de la cuenca. El avance de la forestación ocurrió, fundamentalmente, en la parte media/alta de la cuenca reemplazando terrenos agropecuarios, bosque nativo y matorrales. Los usos agropecuarios experimentaron la mayor pérdida de superficie superando las 20 mil hectáreas, le sigue el bosque nativo con una pérdida de aproximadamente 13 mil hectáreas, y la cobertura de matorrales con una disminución de casi nueve mil hectáreas (Cuadro No 2 y Figura No 3 ).

Figura No 2

Cambio de usos del suelo, periodo 2000-2008
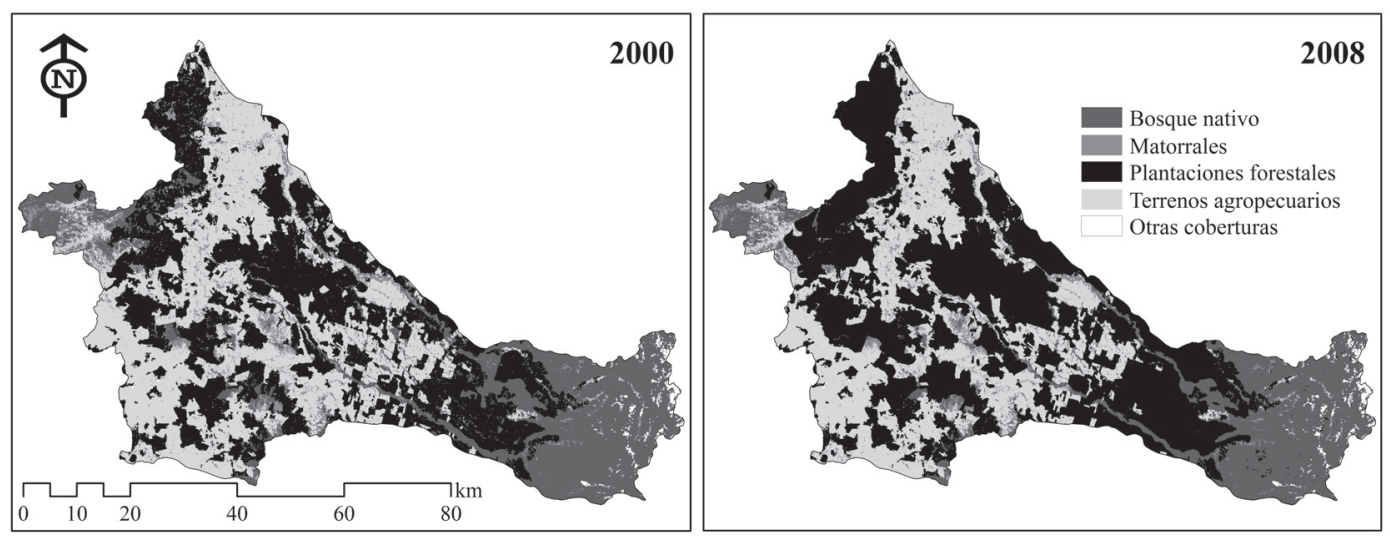

Fuente: Elaboración propia.

Cuadro No 2

Cambios en la superficie $\left(\mathrm{km}^{2}\right)$ de las principales coberturas del suelo según periodo de análisis.

\begin{tabular}{|l|r|r|r|r|r|}
\hline \multicolumn{1}{|c|}{ Cobertura } & Año 2000 & Año 2008 & Año 2018 & Año 2028 & Año 2038 \\
\hline Bosque nativo & 925,3 & 794,0 & 633,7 & 589,0 & 551,9 \\
Matorrales & 299,0 & 209,8 & 150,6 & 117,6 & 91,1 \\
Plantaciones forestales & 1665,8 & 2092,6 & 2610,6 & 2945,1 & 3213,9 \\
Terrenos agropecuarios & 1409,5 & 1204,3 & 909,4 & 653,9 & 449,2 \\
Otras coberturas & 40,2 & 39,1 & 35,6 & 34,3 & 33,7 \\
Total & 4339,8 & 4339,8 & 4339,8 & 4339,8 & 4339,8 \\
\hline
\end{tabular}

Nota: Coberturas de suelo año 2000 y 2008 obtenida a partir de imágenes satelitales. Cobertura suelo años 2018, 2028 y 2038 obtenida a través de la simulación de avance de plantaciones forestales Fuente: Elaboración propia. 
Figura No 3

Variación temporal de las principales coberturas de suelo

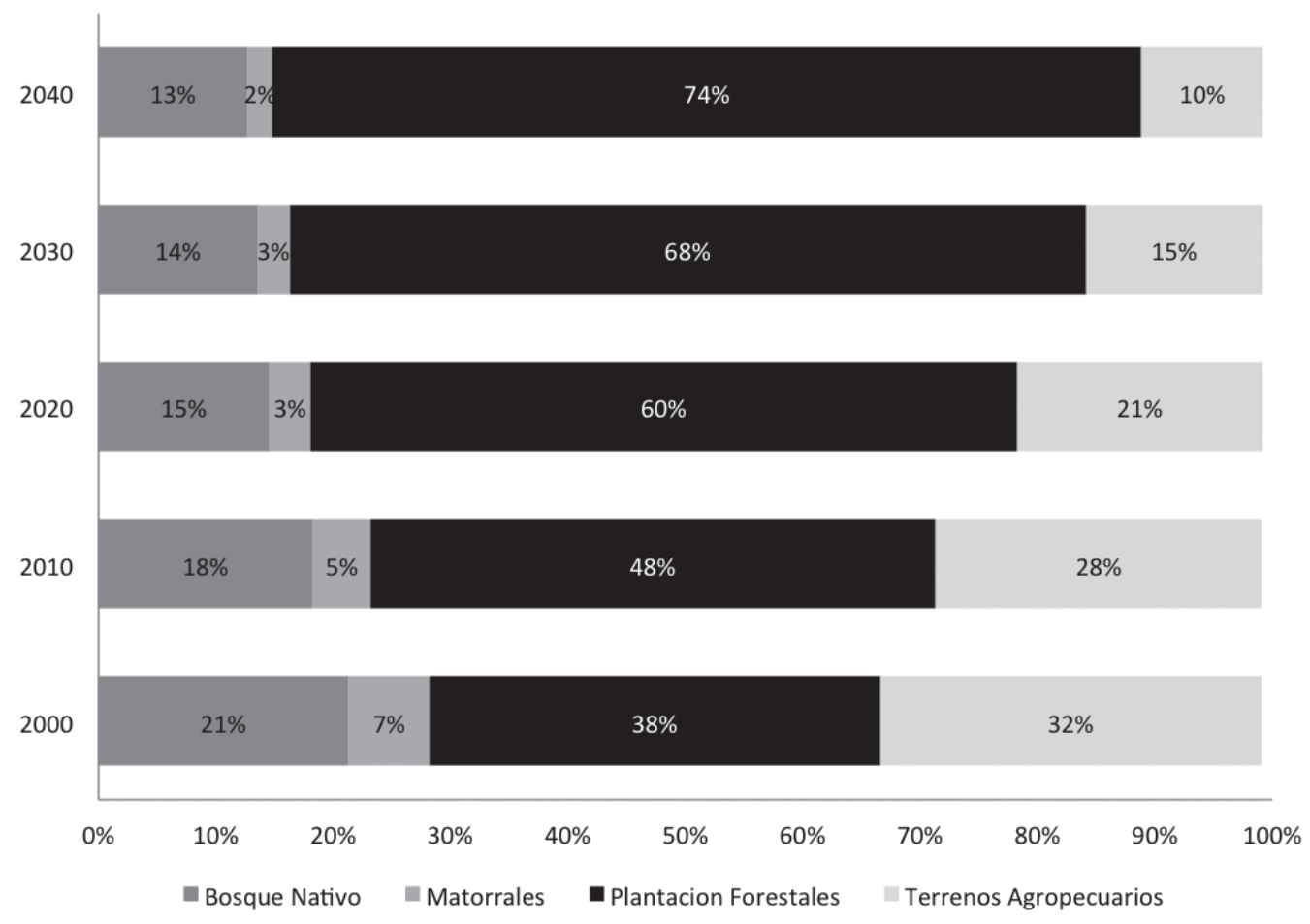

Fuente: Elaboración propia.

El modelo de regresión logística permitió relacionar los factores que determinan el avance de las plantaciones forestales, reproducir adecuadamente su dinámica espacial y generar futuros escenarios de desarrollo forestal. De esta manera, según el patrón espacial de la expansión forestal observado en el periodo 2000-2008, y formalizado a través de la modelación espacialmente explicita, el avance de las plantaciones forestales se encuentra fuertemente determinado por la topografía, distancia a caminos, presencia de plantaciones forestales previamente establecidas, presencia de bosque nativo y tamaño de propiedad. En efecto, el signo de los parámetros de elevación y pendiente en la ecuación del modelo indica que topografías elevadas e inclinadas restringen, en cierta medida, el establecimiento de plantaciones forestales. Por otra parte, los parámetros de distancia demuestran que la actividad forestal se desarrolla cerca de plantaciones previamente establecidas debido a que estas áreas presentan facilidades de infraestructura básica tales como caminos y centros de acopio. Así mismo, la cercanía a áreas cubiertas de bosque nativo promueven la expansión forestal ya que ofrecen condiciones de sitio favorables para su establecimiento; principalmente, suelo de aptitud preferentemente forestal. El tamaño de la propiedad también es una variable que determina el desarrollo forestal promoviendo su establecimiento en sectores rurales dominados por pequeña y mediana propiedad donde la actividad agrícola no ofrece buenas oportunidades económicas (Cuadro No 3 ). 
Cuadro No 3

Parámetros del modelo de regresión logística ajustado para la expansión forestal $\left({ }^{* *}=P<0,01\right)$

\begin{tabular}{|l|r|r|r|r|}
\hline \multicolumn{1}{|c|}{ Variables } & \multicolumn{1}{c|}{$\beta(\mathrm{i})$} & \multicolumn{1}{c|}{ Error estándar } & Wald a) & \multicolumn{1}{c|}{$P$} \\
\hline Elevación & $-0,00283$ & 0,000151 & 353,02 & $* *$ \\
Distancia a bosque nativo & $-0,00097$ & 0,000058 & 283,27 & $* *$ \\
Presencia de plantaciones forestales & 4,55952 & 0,375919 & 147,11 & $* *$ \\
Distancia a plantaciones forestales & $-0,00004$ & 0,000004 & 125,70 & $* *$ \\
Presencia de bosque nativo & 1,80870 & 0,196294 & 84,90 & $* *$ \\
Pendiente & $-0,01825$ & 0,002389 & 58,31 & $* *$ \\
Distancia a caminos & $-0,00003$ & 0,000004 & 41,87 & $* *$ \\
Densidad de caminos & $-23,12424$ & 3,767705 & 37,67 & $* *$ \\
Tamaño de propiedad & $-0,00001$ & 0,000001 & 35,38 & $* *$ \\
Constante & $-0,38950$ & 0,161903 & 5,79 & $* *$ \\
\hline
\end{tabular}

a) La prueba de Wald fue usado para evaluar la significancia estadística de cada coeficiente $(\beta)$ en el modelo.

Fuente: Elaboración propia.

A partir del modelo de Regresión Logística se simularon tres escenarios de expansión forestal proyectados para los próximos 30 años. Aunque es posible tener una representación continua del avance de las plantaciones en la cuenca, la Figura No 4 muestra los resultados para los años 2018, 2028 y 2038. Los escenarios suponen que las condiciones o variables que determinan y promueven el establecimiento de nuevas plantaciones forestales son las mismas que operaron en el periodo de ajuste del modelo; estos es, el avance ocurrido entre 2000 y 2008. De esta manera, para el año 2018 la superficie plantada podría aumentar en un 25\% respecto al año 2008; es decir, aproximadamente 52 mil hectáreas. Para el año 2038 las plantaciones alcanzarían una superficie de 112 mil hectáreas; es decir, casi el doble de la superficie registrada el 2008, pasando a ser la cobertura dominante en la cuenca. En sentido contrario, el escenario simulado para el 2018 muestra una disminución de 30 mil hectáreas (24\%) de terrenos agropecuarios y de 16 mil hectáreas (20\%) de bosque nativo. Así mismo, para el 2038 los terrenos agropecuarios disminuirán en un 63\% (755 mil hectáreas) y el bosque nativo en un 30"\% (242 mil hectáreas) respecto al 2008, reduciendo notablemente su proporción en relación a la superficie de la cuenca (Cuadro $N^{\circ} 2$ y Figura $N^{\circ} 3$ ).

\section{Efectos hidrológicos de futuros escenarios de expansión forestal}

Los resultados de la modelación hidrológica fueron evaluados comparando los caudales medios mensuales simulados versus los observados en las estaciones fluviométricas de Renaico, Mininco y Malleco. El principal problema detectado fue la subestimación de los caudales punta para las tres estaciones usadas en el proceso de validación (Figura No 5). Sin embargo, de acuerdo a los índices de eficiencia de Nash-Sutcliffe (EF), el coeficiente de determinación $\left(R^{2}\right)$ y el porcentaje de desviación (PBIAS), el modelo reproduce satisfactoriamente el régimen hidrológico de 
las tres subcuencas asociada a cada estación. Esto permitió simular adecuadamente el efecto hidrológico generado por los escenarios de expansión forestal (Cuadro № 4). Con los parámetros del modelos hidrológico calibrado y validado, se simularon los caudales medios mensuales para los periodos 2015-2020, 2025-2030 y 2035-2040 usando los escenarios de expansión forestal de los años 2018, 2028 y 2038, respectivamente. Estos datos fueron contrastados con los caudales medios mensuales de verano e invierno obtenido directamente de las estaciones fluviométricas para el periodo base 2005-2010 (Cuadro No 5).

Figura $N^{\circ} 4$

Escenarios futuros de expansión forestal.

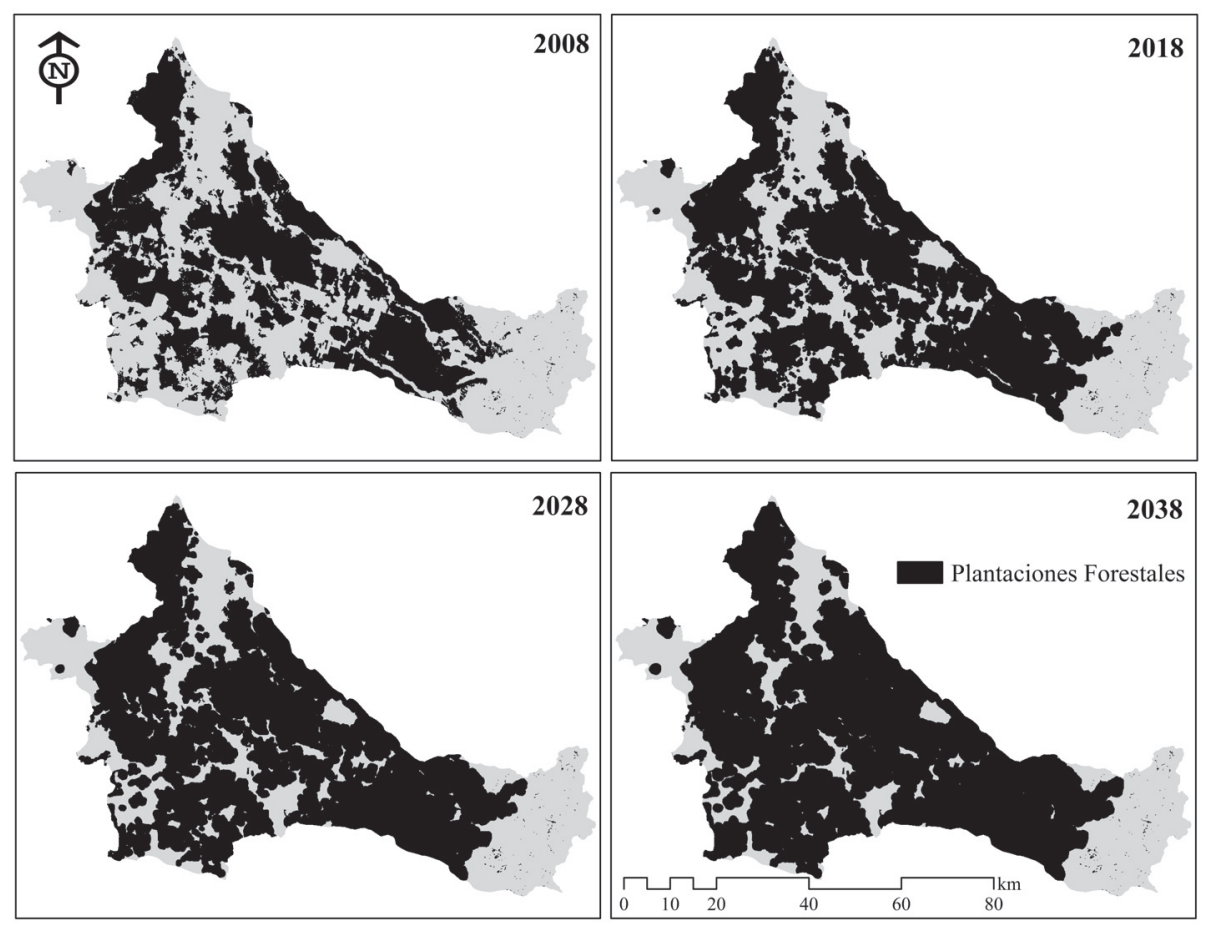

Fuente: Elaboración propia.

Cuadro No 4

Índice de eficiencia de Nash-Sutcliffe (EF), valor absoluto del porcentaje de desviación (PBIAS) y coeficiente de determinación $\left(R^{2}\right)$ calculados para el periodo de validación 2002-2008

\begin{tabular}{|c|c|c|c|}
\hline Subcuencas & $E F$ & $\mathrm{R}^{2}$ & PBIAS \\
\hline Renaico & 0,91 & 0,88 & 4,067 \\
\hline Mininco & 0,87 & 0,80 & 7,95 \\
\hline Malleco & 0,85 & 0,78 & 6,63 \\
\hline
\end{tabular}

Fuente: Elaboración propia. 
Figura No 5

Caudales observados (línea negra) y simulados (línea gris) durante periodo de validación 2002-2008 en las estaciones Renaico (A), Mininco (B) y Malleco (C)
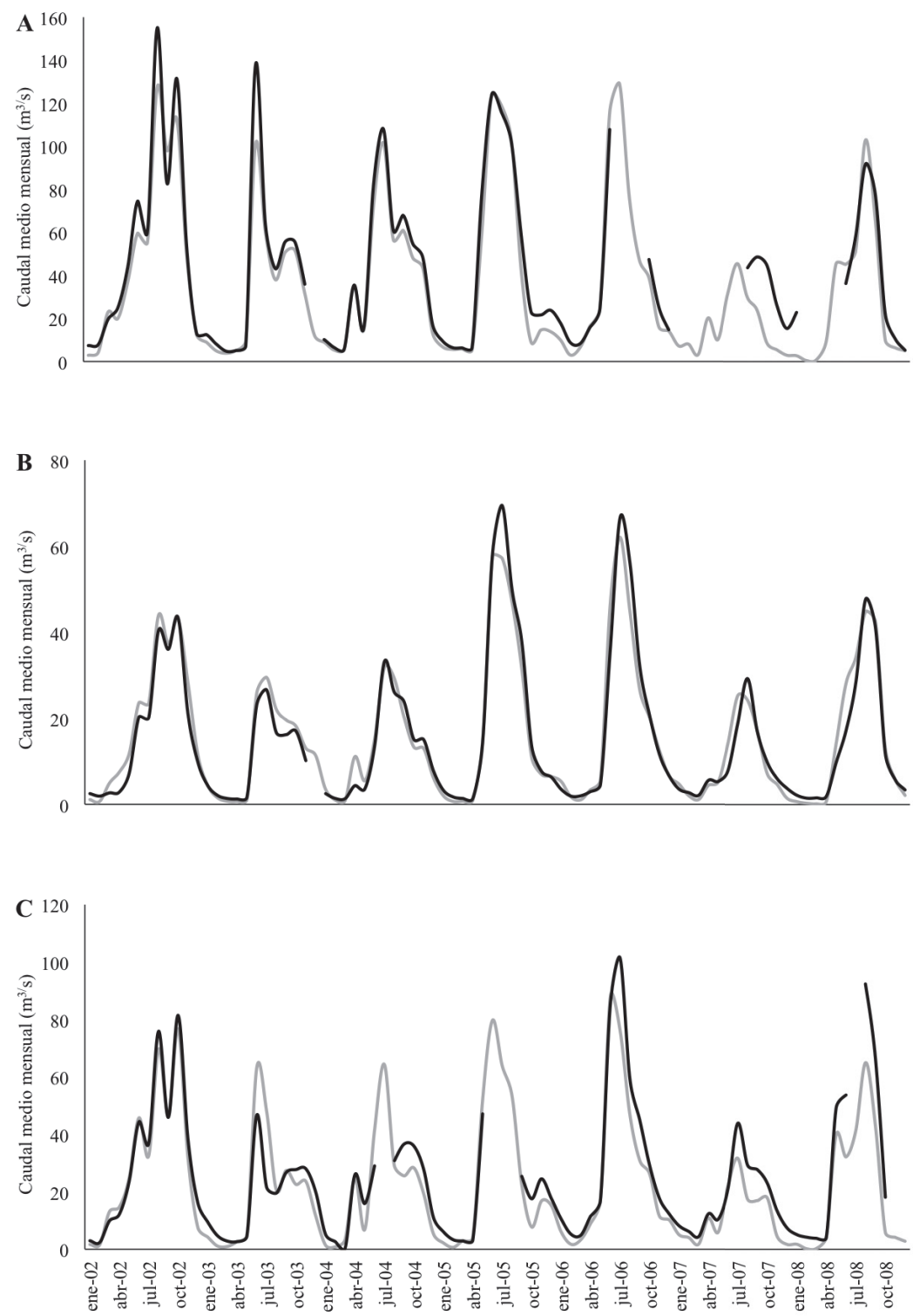

Fuente: Elaboración propia.

Las simulaciones muestran cambios importantes en el caudal medio mensual tanto para la época de verano como para el invierno. Las mayores variaciones de caudal ocurrirían en la subcuenca Malleco debido a un fuerte aumento de las plantaciones forestales y, en consecuencia, a la pérdida de bosque nativo y terrenos agropecuarios. En efecto, para el período 2015-2020 (escenario forestal 2018) se observa una reducción del 55\% de los caudales de verano la que 
incrementaría en la medida que las plantaciones avanzan hacia el período 2035-2040 (escenario forestal 2038) alcanzado una disminución de hasta $60 \%$ respecto a periodo base. Las subcuencas de Renaico y Mininco presentan variaciones más moderadas respecto al periodo base debido a que en la actualidad las plantaciones forestales ocupan una mayor proporción de sus superficies $y$, en consecuencia, el avance de la frontera forestal es menor en tanto las áreas aptas para su establecimiento ya se encuentran cubiertas. Los cambios en los caudales de invierno son sustancialmente menores respecto al periodo base, disminuyendo entre $11 \%$ y $38 \%$ dependiendo de la cuenca y de los escenarios de expansión forestal simulados (Cuadro No 5 y Figura No 6).

Cuadro No 5

Caudales medios mensuales $\left(\mathrm{m}^{3} \mathrm{~s}-1\right)$ de verano e invierno según periodos de análisis

\begin{tabular}{|l|l|r|r|r|r|}
\hline \multirow{2}{*}{ Subcuencas } & Estación & \multicolumn{1}{|c|}{$\begin{array}{c}\text { Periodo } \\
\text { 2005-2010 }\end{array}$} & $\begin{array}{c}\text { Periodo } \\
2015-2020\end{array}$ & $\begin{array}{c}\text { Periodo } \\
2025-2030\end{array}$ & $\begin{array}{c}\text { Periodo } \\
2035-2040\end{array}$ \\
\hline \multirow{2}{*}{ Renaico } & Verano & 8,39 & 5,55 & 5,15 & 4,83 \\
& Invierno & 72,94 & 64,89 & 59,49 & 55,59 \\
\multirow{2}{*}{ Mininco } & Verano & 2,50 & 1,97 & 1,91 & 1,73 \\
\multirow{3}{*}{ Malleco } & Invierno & 38.93 & 33,58 & 31,08 & 29,19 \\
& Verano & 5,20 & 2,32 & 2,18 & 2,03 \\
& Invierno & 53,54 & 38,26 & 35,06 & 32,74 \\
\hline
\end{tabular}

Fuente: Elaboración propia.

Figura No 6

Variación porcentual de los caudales de verano (barra negra) e invierno (barra gris) entre el periodo base y los periodos simulados según los escenarios de expansión forestal.

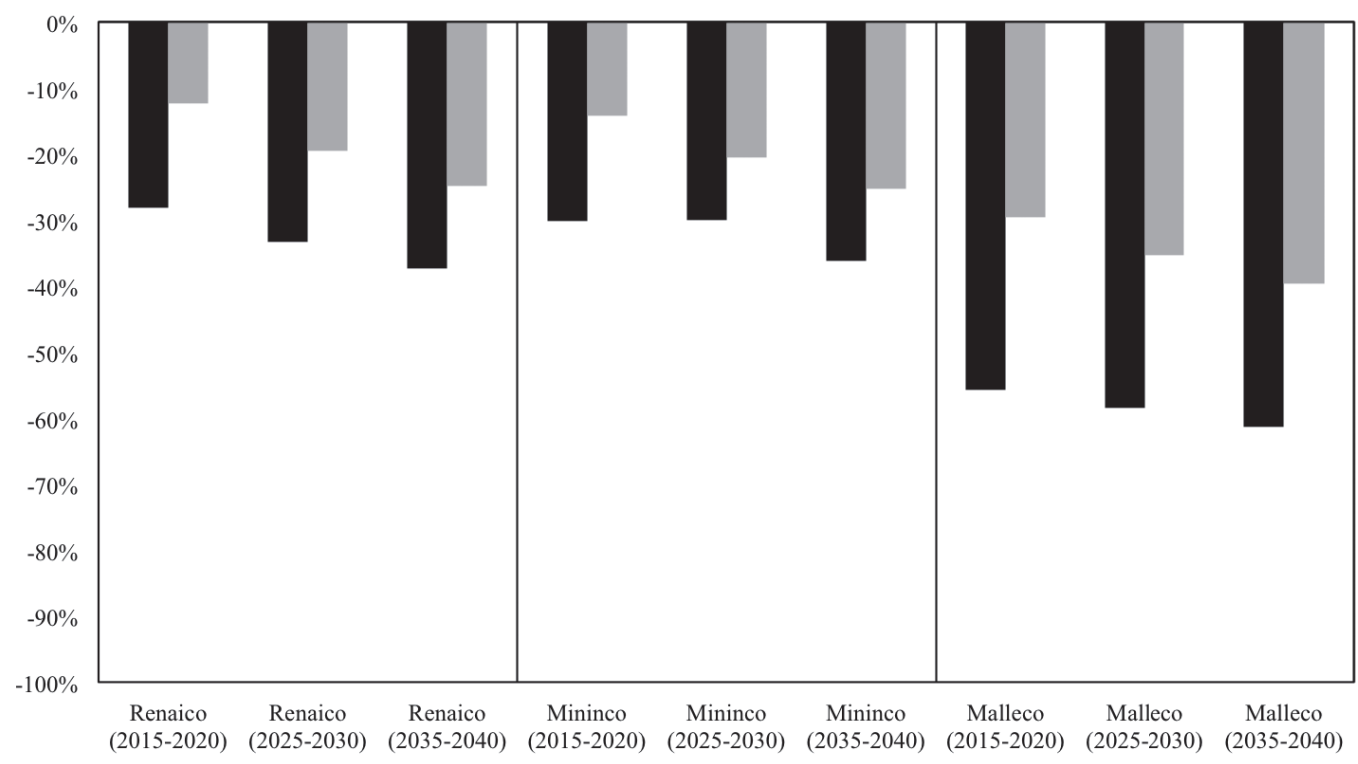

Fuente: Elaboración propia. 
Los resultados de la validación demuestran que el modelo fue capaz de reproducir la hidrología de la cuenca satisfactoriamente y, en consecuencia, es apropiado para evaluar la respuesta hidrológica de escenarios futuros de expansión forestal (Stehr et al., 2010). La tendencia a la disminución de los caudales ante escenarios de aumento de la cobertura forestal es, en general, consistente con lo reportado por otros autores (Bosch \& Hewlett, 1982, Sahín et al., 1996, Calder et al., 1997, Klöcking \& Haberlandt, 2002, Eckhardt et al., 2003, Farley et al., 2005, Huber et al., 2008, Little et al., 2009, Lara et al., 2009, Iroumé \& Palacios, 2013). En efecto, en el clásico estudio de Bosch y Hewlett (1982) se observa la misma tendencia al analizar los resultados de 94 cuencas experimentales de pequeña escala distribuidas en todo el mundo. Estos autores concluyen que el aumento de la cobertura forestal provoca reducciones significativas en los caudales de salida. Sahín et al. (1996), a través del análisis de 145 cuencas -incluidas las analizadas por Bosch y Hewlett (1982)-, concluyen también que cuencas parcial o totalmente deforestadas registran un aumento de los caudales; por el contrario, la reforestación de áreas abiertas conducen a una disminución de los mismos. Las simulaciones hechas por Eckhardt et al. (2003) sobre una cuenca artificial confirman estas tendencias; la conversión de suelos arados en terrenos boscosos reflejan cambios en la escorrentía, recarga de agua subterránea y flujo superficial concentrado cuyo nivel de significancia depende de las proporciones en que estos cambios ocurren. Iroumé y Palacios (2013), detectaron importantes reducciones de caudal en grandes cuencas de régimen pluvial localizadas en el centro-sur de Chile toda vez que el incremento neto del área forestada fue mayor al $16 \%$ del área total de la cuenca.

La tendencia a la disminución de los caudales debido a la expansión forestal es notoriamente más importante en la época de estiaje. Según las simulaciones, las reducciones en los caudales de verano respecto al periodo base son, en algunos caso, el doble más altas que las producidas en invierno. Estas tendencias también son observadas en estudio de pequeñas cuencas del sur de Chile demostrando que la conversión de bosque nativo por plantaciones forestales provoca un decrecimiento de los caudales especialmente en verano (Otero et al., 1994, Lara et al., 2009). Lara et al. (2009) reportan una reducción de $20,4 \%$ de la escorrentía de verano por cada $10 \%$ de incremento de las plantaciones forestales en seis pequeñas cuencas. Por su parte, Little et al. (2009) observa la misma tendencia para cuencas de meso escala donde el efecto del aumento de las plantaciones sobre el régimen hidrológico es significativamente más importante en verano. Ellos observaron que el reemplazo de bosques nativos por plantaciones forestales de rápido crecimiento ha provocado una caída de los caudales de verano en un $42,7 \%$ y un $31,9 \%$ en dos cuencas de gran tamaño, respectivamente (Little et al., 2009; Little y Lara, 2010).

Muchos autores coinciden en que el principal efecto que tienen las plantaciones forestales sobre el balance hídrico se debe a la cantidad de precipitación que es retenida en el dosel y cambios en los procesos de evapotranspiración reduciendo, de esta manera, la escorrentía superficial, los caudales de descarga y, finalmente, la producción hídrica de la cuenca (e.g. Bosch \& Hewlett, 1982; Calder, 1993; Putuhena \& Cordery, 2000, Iroumé \& Palacios, 2013). En efecto, la forestación modifica la cantidad de agua involucrada tanto en la evaporación desde suelo como en la transpiración de los árboles. Este valor varía dependiendo del total de agua disponible en el suelo, las características de las plantaciones y las condiciones meteorológicas (Putuhena \& Cordery, 2000). Además, la percolación de agua en el suelo también es alterada por la forestación debido a que es regulada por variables que influyen sobre la cantidad de agua que alcanza la superficie y la evapotranspiración neta (Putuhena \& Cordery, 2000). Huber et al. (2008) encuentran importantes diferencias en los valores de intercepción, evapotranspiración y percolación al 
comparar diferentes parcelas experimentales cubiertas por plantaciones de pino radia ubicadas en el centro-sur de Chile, entre los $35^{\circ}$ y $40^{\circ}$ latitud sur. Ellos muestran que la interceptación de la lluvia incrementa de sur a norte desde $15 \%$ a $40 \%$. En el mismo sentido, la evaporación neta (transpiración y evaporación del suelo) varía desde 32"\% a 55"\%. Los valores de percolación son mínimos en los sitios del norte (5\%) y máximos en los del sur (53\%). Este proceso es regulado por el régimen de precipitaciones y por la capacidad de retención de humedad del suelo. En las cuencas más septentrionales el agua caída es casi completamente evapotranspirada alcanzando valores superiores al 75"\% (Huber et al., 2008).

Los actuales problemas de disponibilidad de agua se agudizarán en la medida que la demanda creciente por recursos hídricos se enfrente a futuros escenarios de aumento de temperaturas y disminución de precipitaciones (CONAMA-DGF 2006). En efecto, las proyecciones climática para el centro-sur de Chile en las próximas décadas (2011-2030) estiman que las temperaturas aumentarán entre 0,5 y $1,5^{\circ} \mathrm{C}$ y las precipitaciones disminuirán entre 5 y $20 \%$ (Rojas, 2012a y 2012b). En este contexto, los modelos hidrológicos tienen la capacidad de predecir los efectos acumulativos y/o sinérgicos del cambio climático y cambio de uso del suelo convirtiéndose en una herramienta apropiada para la toma decisiones anticipadas y la implementación de medidas preventivas ante escenarios de escases hídrica (Bronstert et al., 2002). En el ámbito forestal, estos modelos permiten evaluar distintas prácticas de manejo considerando no solo escenarios de plantaciones plenamente establecidas sino también distintas edades, densidades, especies y periodos de rotación. De la misma manera, estas herramientas de análisis tienen la capacidad de simular los efectos de diversas medidas de conservación orientadas a la protección de los recursos hídricos tales como planes de reforestación y restauración de riberas.

\section{Conclusiones}

Este estudio releva la utilidad que poseen los modelos hidrológicos, acoplados a modelos de cambio de uso del suelo espacialmente explícitos, para someter a prueba hipótesis relacionadas con los potenciales efectos de distintas intervención del territorio en cuencas de meso o gran escala. Similares métodos pueden ser usados para apoyar la toma decisiones en el ámbito de la gestión integrada de cuencas hidrográficas. Así por ejemplo, los modelos hidrológicos pueden ser utilizados para evaluar los alcances de programas de incentivo a la inversión, manejo y/o conservación de recursos hídricos. Los resultados del proceso de validación prueban que el modelo empleado en este caso de estudio es capaz de reproducir adecuadamente la respuesta hidrológica de distintos escenarios de expansión forestal. En este sentido, las simulaciones del régimen de caudal generadas son una primera aproximación a los potenciales efectos que el aumento de la cobertura de plantaciones provocaría sobre la disponibilidad de agua en la cuenca, sin considerar futuros cambios en el patrón de precipitaciones. En efecto, las simulaciones muestran una tendencia a la disminución de los caudales lo cual es especialmente preocupante en la época de estiaje donde el caudal se reduce a más del $50 \%$ respecto al período base. Esto es consistente con las tendencias reportadas por otros autores en cuencas de pequeña y meso escala localizadas en el centro-sur de Chile. 


\section{Referencias bibliográficas}

AGUAYO, M.; PAUCHARD, A.; AZÓCAR, G. y PARRA, O. Cambio del uso del suelo en el centro sur de Chile a fines del siglo XX. Entendiendo la dinámica espacial y temporal del paisaje. Revista Chilena de Historia Natural, 2009, Vol. 82, p. 361-374.

AGUAYO, M.; WIEGAND, T.; AZÓCAR, G.; WIEGAND, K. \& VEGA, C. Revealing the driving forces of midcities urban growth patterns using spatial modeling: a case study of Los Ángeles, Chile. Ecology and Society, 2007, Vol. 12, No 1, p. 13. Disponible en Internet: http://www.ecologyandsociety.org/ vol12/iss1/art13/

AGUAYO, M. El cambio de uso de suelo en el centro-sur de Chile. Comprendiendo su dinámica a través de la modelación espacialmente explícita y explorando sus efectos ambientales en una cuenca hidrológica de mesoescala. Concepción: Tesis Doctorado en Ciencias Ambientales, Universidad de Concepción, 2008.

ALTAMIRANO, A.; APLIN, P.; MIRANDA, A.; CAYUELA, L.; ALGAR, A. \& FIELD, R. High rates of forest loss and turnover obscured by classical landscape measures. Applied Geography, 2013, Vol. 40, p. 199-211.

ARNOLD, J. G. \& FOHRER, N. SWAT2000: current capabilities and research opportunities in applied watershed modelling. Hydrological Processes, 2005, Vol. 19, No 3, p. 563-572.

ARNOLD, J.G.; SRINIVASAN, R.; MUTTIAH, R.S. \& WILLIAMS, J. Large area hydrologic modeling and assessment - Part 1: Model development. Journal Of The American Water Resources Association, 1998, Vol. 34, No 1, p. 73-89.

BOSCH, J.M. \& HEWLETT, J.D. A review of catchment experiments to determine the effect of vegetation changes on water yield and evapotranspiration. Journal of Hydrology, 1982, Vol. 55, p. 3-23.

BRONSTERT, A.; NIEHOFF, D. \& BURGER, G. Effects of climate and land-use change on storm runoff generation: present knowledge and modelling capabilities. Hydrological Processes, 2002, Vol. 16, No 2, p. 509-529.

BROWN, A.E.; ZHANG, L.; MCMAHON, T.A.; WESTERN, A.W. \& VERTESSY, R. A review of paired catchment studies for determining changes in water yield resulting from alterations in vegetation. Journal of Hydrology, 2005, Vol. 310, p. 28-61.

CALDER, I.R.; ROSIER, P.T.W.; PRASANNA, K.T. \& PARAMESWARAPPA, S. Eucalyptus water use greater than rainfall input a possible explanation from southern India hydrological and earth system. Hydrology and Earth System Sciences, 1997, Vol. 1, No 2, p. 249-256.

CALDER, I. R. Hydrologic Effects of Land-Use Change. En: MAIDMENT, D.R. (editors). Handbook of Hydrology. New York: McGraw-Hill, 1993. 
CIREN. Estudio agrológico VIII Región. Descripción de suelos, materiales y símbolos. Tomos 1 y 2. Santiago de Chile: CIREN CORFO, 1999a.

CIREN. Estudio agrológico IX Región. Descripción de suelos, materiales y símbolos. Tomos 1 y 2. Santiago de Chile: CIREN CORFO, 1999b.

CONAMA-DGF. Estudio de la variabilidad climática en Chile para el siglo XXI. Santiago, Chile. Santiago de Chile: Comisión Nacional del Medio Ambiente / Departamento de Geofísica, Facultad de Ciencias Físicas y Matemáticas, Universidad de Chile, 2006.

CHUVIECO, E. Fundamentos de teledetección espacial. Madrid: RIALP S.A., 1996.

ECHEVERRÍA, C.; HUBER, A. \& TABERLET, F. Estudio comparativo de los componentes del balance hídrico en un bosque nativo y una pradera en el sur de Chile. Bosque, 2008, Vol. 28, № 3, p. 271-280.

ECKHARDT, K.; BREUER, L. \& FREDE, H.G. Parameter uncertainty and the significance of simulated land use change effects. Journal of Hydrology, 2003, Vol. 273, No 1-4, p.164-176.

FARLEY, K.A.; JOBBAGY, E.G. \& JACKSON, R.B. Effects of afforestation on water yield: a global synthesis with implications for policy. Global Change Biology, 2005, Vol. 11, № 10, p.1565-1576.

FOHRER, N.; HAVERKAMP, S.; ECKHARDT, K \& FREDE, H. G. Hydrologic response to land use changes on the catchment scale. Physics And Chemistry of The Earth Part B-Hydrology Oceans and Atmosphere, 2001, Vol. 26, No 7-8, p. 577-582.

FOLEY, J.A.; DEFRIES, R.; ASNER, G.P.; BARFORD, C.; BONAN, G.; CARPENTER, S.P.; CHAPIN, F.S.; COE, M.T.; DAILY, G.C.; GIBBS, H.K.; HELKOWSKI, J.H.; HOLLOWAY, T.; HOWARD, E.A.; KUCHARIK, C.J.; MONFREDA, C.; PATZ, J.A.; PRENTICE, I.C.; RAMANKUTTY, N. \& SNYDER, P.K. Global consequences of land use. Science, 2005, Vol. 309, No 5734, p. 570-574.

HUBER, A.; IROUMÉ, A. \& BATHURST, J. Effect of Pinus radiata plantation on water balance in Chile. Hydrological Process, 2008, Vol. 22, p.142-148.

IROUMÉ, A. \& PALACIOS, H. Afforestation and changes in forest composition affect runoff in large river basins with pluvial regime and Mediterranean climate, Chile. Journal of Hydrology, 2013, Vol. 505, p.113-125.

IROUME, A.; HUBER, A. \& SCHULZ, K. Summer flows in experimental catchments with different forest covers, Chile. Journal of Hydrology, 2005, Vol. 300, № 1-4, p. 300-313.

KLOCKING, B. \& HABERLANDT, U. Impact of land use changes on water dynamics-a case study in temperate meso and macroscale river basins. Physics and Chemistry of the Earth, 2002, Vol. 27, No 9-10, p. 619-629.

KLOCKING, B.; STROBL, B.; KNOBLAUCH, S.; MAIER, U.; PFUTZNER, B. \& GERICKE, A. Development and allocation of land-use scenarios in agriculture for hydrological impact studies. Physics and Chemistry of the Earth, 2003, Vol. 28, № 33-36, p. 1311-1321. 
LARA, A.; SOLARI, M.; PRIETO, M. \& PEÑA, M. Reconstrucción de la cobertura de la vegetación y uso del suelo hacia 1550 y sus cambios a 2007 en la ecorregión de los bosques valdivianos lluviosos de Chile (35 - $43^{\circ} 30$ S). Bosque, 2012, Vol. 33, No 1, p. 13-23.

LARA, A.; LITTLE, C.; URRUTIA, R.; MCPHEE, J.; ÁLVAREZ-GARRETÓN, C.; OYARZÚN, C.; SOTO, D.; DONOSO, P.; NAHUELHUAL, L.; PINO, M. \& ARISMENDI, I. Assessment of ecosystem services as an opportunity for the conservation and management of native forests in Chile. Forest Ecology and Management, 2009, Vol. 258, p. 415-424.

LITTLE, C.; LARA, A.; MCPHEE, J. \& URRUTIA, R. Revealing the impact of forest exotic plantations on water yield in large scale watersheds in South-Central Chile. Journal of Hydrology, 2009, Vol. 374, p.162-170.

LITTLE, C. \& LARA, A. Restauración ecológica para aumentar la provisión de agua como un servicio ecosistémico en cuencas forestales del centro-sur de Chile. Bosque, 2010, Vol. 31, № 3, p. 175-178.

MARK, A.F. \& DICKINSON, K.J.M. Maximizing water yield with indigenous non-forest vegetation: a New Zealand perspective. Frontiers in Ecology and the Environment, 2008. Vol. 6, № 1, p. 25-34.

OTERO, L.; CONTRERAS, A. \& BARRALES, L. Análisis de los efectos ambientales del reemplazo de bosque nativo por plantaciones (efectos sobre cuatro microcuencas en la provincia de Valdivia). Ciencia e Investigación Forestal, 1994, Vol. 8, p. 253-276.

POSTEL, S.L.; DAILY, G.C. \& EHRLICH, P.R. Human appropriation of renewable fresh ater. Science, 1996, Vol. 271, No 5250, p. 785-788.

PUTUHENA, W. \& CORDERY, I. Some hydrological effects of changing forest cover from eucaliptus to Pinus radiata. Agricultural and Forest Meteorology, 2000, Vol. 100, p.59-72.

ROJAS, M. Estado del arte de modelos para la investigación del calentamiento global. Santiago de Chile: Informe para Opciones de Mitigación para enfrentar el Cambio Climático, MAPS Chile, 2012a.

ROJAS, M. Proyecciones climáticas futuras y análisis de incertidumbre. En La Economía del cambio climático en Chile. Santiago de Chile: Colección Documentos de proyectos, CEPAL, 2012b.

SAHIN, V. \& HALL, M.J. The effects of afforestation and deforestation on water yields. Journal of Hydrology, 1996, Vol. 178, No 1-4, p. 293-309.

SANGREY, D.A.; HARROP-WILLIAMS, K.O. \& KLAIBER, J.A. Predicting ground-water response to precipitation. ASCE J. Geotech. Eng., 1984, Vol. 110, No 7, p. 957-975.

STEHR, A.; AGUAYO, M.; LINK, O.; PARRA, O.; ROMERO, F. \& ALCAYAGA, H. Modelling the hydrologic response of a mesoscale Andean watershed to changes in land use patterns for environmental planning. Hydrology and Earth System Sciences, 2010, Vol. 14, No 10, p.1963-1977. 
VAN LIEW, M.W.; ARNOLD, J. G. \& BOSCH, D.D. Problems and potential of autocalibrating a hydrologic model. Transactions of the ASAE, 2005, Vol. 48, No 3, p.1025-1040.

VENETIS, C. A study of the recession of unconfined aquifers. Bull. Int Assoc. Sci. Hydrol, 1969, Vol. $14, N^{\circ} 4$, p. 119-125.

WILK, J.; ANDERSSON, L. \& PLERMKAMON, V. Hydrological impacts of forest conversion to agriculture in a large river basin in northeast Thailand. Hydrological Processes, 2001, Vol. 15, No 14, p. 2729-2748. 